\title{
Predictors and outcome of complete removal of colorectal cancer with synchronous lung metastases
}

\author{
HIROAKI NOZAWA $^{1}$, JUNICHIRO TANAKA ${ }^{1}$, TAKESHI NISHIKAWA ${ }^{1}$, TOSHIAKI TANAKA ${ }^{1}$, \\ TOMOMICHI KIYOMATSU ${ }^{1}$, KAZUSHIGE KAWAI ${ }^{1}$, KEISUKE HATA ${ }^{1}$, SHINSUKE KAZAMA ${ }^{1}$, \\ HIRONORI YAMAGUCHI $^{1}$, SOICHIRO ISHIHARA ${ }^{1}$, EIJI SUNAMI ${ }^{1}$, JOJI KITAYAMA ${ }^{1}$, \\ JUN NAKAJIMA $^{2}$, NORIHIRO KOKUDO ${ }^{3}$ and TOSHIAKI WATANABE ${ }^{1}$ \\ Departments of ${ }^{1}$ Surgical Oncology, ${ }^{2}$ Thoracic Surgery and ${ }^{3}$ Hepatobiliary and Pancreatic Surgery, \\ The University of Tokyo, Tokyo 113-8655, Japan
}

Received December 24, 2014; Accepted April 8, 2015

DOI: $10.3892 / \mathrm{mco} .2015 .599$

\begin{abstract}
The prognosis-improving effect of radical surgery has been demonstrated in patients with colorectal cancer (CRC) with liver metastases. However, few studies have examined the effectiveness of treatments for CRC with metastases in organs other than the liver. The aim of the present study was to evaluate the outcome of surgical treatment for CRC with lung metastases. The study retrospectively examined 57 primary CRC patients ( 28 men, median age of 65 years) with synchronous lung metastases who underwent surgery between 2003 and 2012. Data such as clinicopathological parameters, metastasized organs, and the details of surgery, recurrence and survival periods were extracted and analyzed. Curative resection was performed in 10 patients ('curative group'). Primary tumors were resected without metastasectomy in 37 patients ('non-curative group'), whereas 10 underwent stoma surgery ('stoma group'). All the metastasized lesions were confined to the lung and liver in the curative group. By contrast, $43 \%$ of the non-curative/stoma groups had metastases in organs other than the lung and liver. Multivariate analyses indicated the absence of extrahepatic metastases as the only predictor of curative resection in CRC patients with lung metastases. The 3 -year overall survival rates for the curative, non-curative and stoma groups were estimated as 74, 20 and 17\%, respectively $(\mathrm{P}=0.0007)$. In conclusion, curative resection was possible in CRC patients with lung metastases if other disseminated lesions were limited to the liver and this treatment resulted in a longer survival time. Furthermore, palliative resection may contribute to a better prognosis compared to stoma surgery alone in selected cases.
\end{abstract}

Correspondence to: Dr Hiroaki Nozawa, Department of Surgical Oncology, The University of Tokyo, 7-3-1 Hongo, Tokyo 113-8655, Japan

E-mail: hiroanozawa-gi@umin.ac.jp

Key words: colorectal cancer, lung metastasis, curative resection

\section{Introduction}

Colorectal cancer (CRC) is one of the most prevalent malignancies worldwide (1-3). The most common sites of metastases from $\mathrm{CRC}$ are the liver and lung with reported frequencies of 20-70 and 10-20\%, respectively (4). Approximately $25 \%$ of CRC patients were initially diagnosed with these metastases (5). Improvements in clinical outcomes of patients with metastatic CRC have been achieved by upfront chemotherapeutic agents such as cytotoxic drug combinations, including irinotecan, oxaliplatin and 5-fluorouracil, and more recently, biologics targeting vascular endothelial growth factor and/or tyrosine kinase receptors (5). Various types of therapy, such as radiation therapy, radiofrequency ablation and hyperthermia, can be applied in consort with state-of-the-art chemotherapy depending on the metastatic sites (6). Regardless of the advances in these treatment modalities, surgical resection remains the mainstay of treatment for CRC $(4,6)$.

Hepatic resection has been well-established as a treatment for liver metastases from CRC (6). In addition, a recent randomized controlled trial demonstrated the favorable effects of perioperative systemic chemotherapy on postoperative progression-free survival in CRC with liver-limited disease (7). Surgical resection of lung metastases has also gained general acceptance, as increasing literature has suggested that lung metastasectomy may improve the survival rate in selected CRC patients (6). However, few studies have been conducted to determine which CRC patients with lung metastases are capable of undergoing metastasectomy with curative intent.

The aim of the present study was to elucidate the predictive factors for curative resection in CRC patients with synchronous lung metastases. The effects of surgical resection on the prognosis of these patients were also investigated.

\section{Materials and methods}

Patients, treatment and data collection. Patients with primary CRC with synchronous lung metastases who underwent surgery in the Univeristy of Tokyo Hospital (Tokyo, Japan) between January 2003 and December 2012 were subjects in 
the present study. All the patient records regarding age, gender, Eastern Cooperative Oncology Group (ECOG) performance status (PS), preoperative serum carcinoembryonic antigen (CEA) and carbohydrate antigen 19-9 (CA19-9) levels and pathological factors of the primary tumor, pulmonary metastases, hilar lymph node metastases and metastases in other organs were collected. The pathological description was based on the tumor-node-metastasis classification, 7th edition (8). When lung metastases were multiple, the largest diameter was recorded. Based on the type of surgery, patients were divided into curative, non-curative and stoma groups. The 'curative group' included patients who underwent complete resection of primary $\mathrm{CRC}$, as well as metastasectomies for all the metastases. The 'non-curative group' consisted of patients who underwent resection for the primary tumor with metastasized lesions left macroscopically in their bodies. Patients who underwent stoma surgery without tumor resection were classified into the 'stoma group'. In general, resections of lung and other organ metastases were performed regardless of their sizes, number and distribution, as long as the patients met the following criteria: i) Primary CRC should be controllable; ii) all metastases should be potentially resectable; and iii) the patient should be expected to tolerate surgical procedures and to carry out a normal life with the remaining organ functions. Furthermore, perioperative chemotherapy, recurrence and overall survival (OS) were reviewed.

The study was approved by the Ethics Committee of the Univeristy of Tokyo Hospital (Tokyo, Japan).

Statistical analysis. Comparisons of clinicopathological parameters were performed using the Fisher's exact test and Pearson's $\chi^{2}$ test, applying Yates' correction where appropriate. The Wilcoxon rank sum test was applied to assess differences in age and the sizes of the primary tumor and the largest lung metastasis due to their non-parametric nature. OS curves were estimated with the Kaplan-Meier method and analyzed by the log-rank test. Predictive factors for curative resection and prognostic factors for OS were estimated using univariate and multivariate analyses (logistic regression and Cox proportional hazards models, respectively). $\mathrm{P}<0.05$ was considered to indicate a statistically significant difference.

\section{Results}

Baseline characteristics of patients and primary CRC. The total number of patients analyzed in the present study was 57 (28 men; median age, 65 years). Forty-four patients (77\%) had an ECOG PS of 0. Preoperative CEA was elevated in 43 patients $(75 \%)$, whereas CA19-9 was elevated in 27 (47\%). The primary tumor caused an obstruction in 36 cases $(63 \%)$. The distribution of the primary tumor was the right-side of the colon in 11 cases (19\%), left-side of the colon in $17(30 \%)$ and rectum in $29(51 \%)$. Sixteen cases $(28 \%)$ had well-differentiated adenocarcinoma, $34(60 \%)$ had moderately differentiated and $6(11 \%)$ had undifferentiated histology. Among the 47 patients who underwent resection as described below, the median size of the primary tumor was $58 \mathrm{~mm}$ in diameter. The primary tumor invaded through the serosa or further in 23 cases $(49 \%)$. Regional lymph node metastases were detected in 36 cases (77\%), of which 16 were $\mathrm{pN} 2$ or $\mathrm{pN} 3$.
Lung and other metastases. Out of the 57 cases, multiple lung metastases were detected at the time of diagnosis in 48 patients (84\%), with bilateral involvement in 42 (74\%). The maximum size of lung metastases was $10.0 \mathrm{~mm}$ on average. Hilar lymphadenopathy was observed in 6 patients (11\%). Forty-two patients simultaneously had extrapulmonary distant organ metastases, with the liver being the most frequent site (34 cases or $60 \%$ ), followed by distant lymph nodes (14 cases or $25 \%$ ). Peritoneal dissemination and bone and adrenal gland metastases were observed in 8, 4 and 1 cases (14, 7 and 2\%), respectively.

Preoperative chemotherapy and surgery. Preoperative chemotherapy was administered to 6 patients. All received the FOLFOX (5-fluorouracil, leucovorin and oxaliplatin) regimen. Bevacizumab was administered simultaneously with FOLFOX in 1 patient. Another patient received FOLFIRI (5-fluorouracil, leucovorin and irinotecan) as a second-line following the first-line FOLFOX.

Complete resection was performed in 10 patients. The treatment courses of the 'curative group' are depicted in Fig. 1. Four patients had lung-only metastases and 6 manifested synchronous liver metastases; no metastasized organs were found other than the lung and liver. Only 1 patient underwent simultaneous resection for the primary rectal cancer and metastases in the lung and liver. Eight patients underwent two-staged resection for primary CRC and metastasized lesions. Of these, 2 initially underwent metastasectomy for lung lesion(s), followed by colorectal resection. The 6 other patients underwent pulmonary metastasectomy subsequent to resecting the primary tumor with or without hepatectomy (including a case receiving preoperative bevacizumab plus FOLFOX). One patient initially underwent surgery for obstructive sigmoid colon cancer, followed by hepatectomy for liver metastases. A metastatic deposit in the lung and recurrent liver metastases were removed in the third surgery following chemotherapy in this case. Two patients received an approach of thoracotomy for lung metastases in 2003-2004, while 8 had video-assisted thoracoscopic surgery after 2005 . There were 2 lobectomies and 13 wedge resections.

Primary CRC was resected in 37 patients without metastatectomy of some or all metastasized lesion(s) ('non-curative group'). Among these, 3 patients (8\%) received preoperative FOLFOX.

The remaining 10 patients underwent simple colostomy or ileostomy ('stoma group'). FOLFOX was administered before stoma surgery in 1 patient $(10 \%)$.

Only 2 patients in the non-curative group (5\%) experienced surgery-related Clavien-Dindo grade 3 morbidities.

Predictors of curative surgery. Table I compares the clinicopathological parameters between the curative group and others (including non-curative and stoma groups). Patients were significantly older (66.7 years old), whereas obstructive CRC was more common (70\%) in the non-curative/stoma groups compared to the curative group (59.3 years old and $30 \%$, respectively). Both lungs were more likely to be involved in the non-curative/stoma groups ( 81 vs. $40 \%, \mathrm{P}=0.02)$. The frequency of extrapulmonary metastases was likely to be high $(77 \%)$ in the non-curative/stoma groups. Organ involvement 


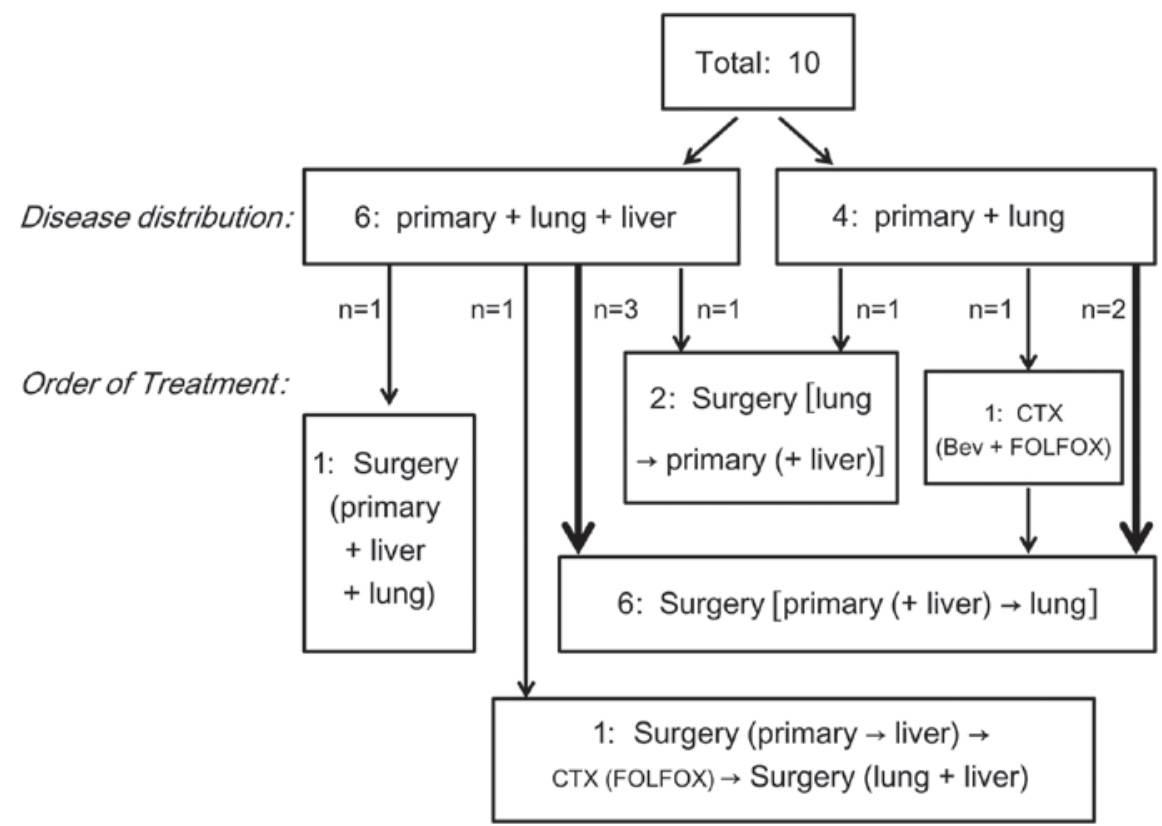

Figure 1. Treatment sequence of 10 colorectal cancer patients with synchronous lung metastases who underwent curative surgery. CTX, chemotherapy; Bev, bevacizumab.

other than the lung and liver was specific to the non-curative/stoma groups (20 patients or $43 \%, \mathrm{P}=0.01)$. No significant intergroup differences were observed in other factors.

The 4 variables (age, obstruction, bilateral lung metastases and extrahepatic disease) were further tested by multivariate analysis (Table II). Logistic regression analysis revealed that only the absence of extrahepatic metastases was a robust, independent predictor of curative surgery in CRC patients with synchronous lung metastases (odds ratio $=1.74 \times 10^{7}, \mathrm{P}=0.011$ ). An age $<65$ years was a marginally significant predictor of curative resection (odds ratio $=6.21, \mathrm{P}=0.052$ ).

Postoperative chemotherapy. In the curative group, 6 patients $(60 \%)$ received adjuvant chemotherapy for a median of 7 months (range, 2-42 months). The chemotherapeutic regimens included 5-fluorouracil plus leucovorin in 3 cases, oral $\mathrm{S}-1$ in 2 and FOLFOX in 1 case.

In the palliative setting, chemotherapy was administered to 37 patients $(79 \%)$ in the non-curative/stoma groups; 7 did not receive any and no information of chemotherapy was available in 3 cases. Six patients $(60 \%)$ in the curative group also received palliative chemotherapy following recurrence.

Therefore, a total of 45 patients (79\%) out of 57 received either adjuvant or palliative chemotherapy or both.

Long-term outcome. Recurrence was observed in $9(90 \%)$ out of 10 patients who underwent curative surgery. The most frequent site of recurrence was the liver ( 6 cases). Three patients with recurrent liver metastases underwent repeated hepatectomy and were alive without evidence of relapse. Recurrent deposits were metachronously detected in the lung in 5 cases. Of these, only 1 patient underwent repeated metastasectomy and was alive without further recurrence. Multiple lymph node metastases in the pelvic wall and mediastinum were treated by radiation and long-term chemotherapy in 1 patient, which resulted in a complete response. By contrast, bone metastases

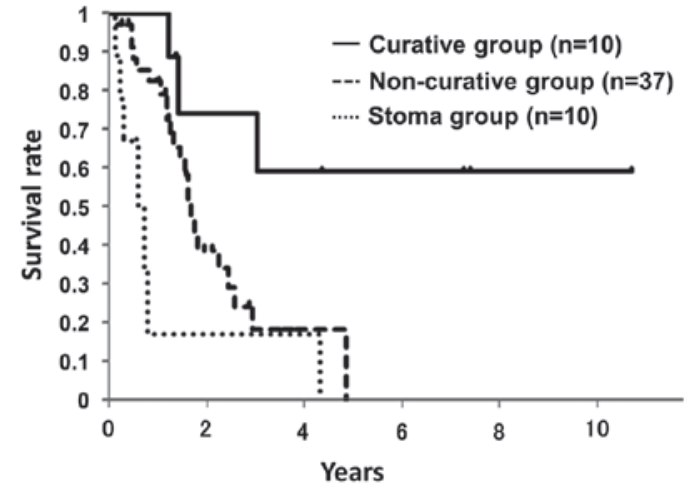

Figure 2. Overall survival rate estimates for colorectal cancer patients with synchronous lung metastases stratified by the type of surgical treatment. Bold line, curative group; dashed line, non-curative group; dotted line, stoma group.

(1 patient) and local recurrence (1 patient) were subjected to systemic palliative chemotherapy.

In the non-curative/stoma groups, 16 patients (34\%) developed further metastases. The distribution of metastases included various organs, such as the lymph nodes (5 cases), liver (4 cases), peritoneum (4 cases), bone (3 cases), brain (3 cases), local (3 cases), ovary (1 case) and adrenal gland (1 case). None of these were surgically resected.

The median follow-up of the entire cohort was 15.4 months. Fig. 2 shows a survival probability curve for each group. The 3 -year survival rate in the curative group (74\%) was markedly higher than that in the non-curative $(20 \%)$ or stoma group (17\%) $(\mathrm{P}=0.018$ and $\mathrm{P}=0.001$, respectively). There was also a significant difference in OS between the non-curative and stoma groups $(\mathrm{P}=0.028)$. The 5-year survival rate for the curative group was estimated as $59 \%$, whereas none in the non-curative/stoma groups survived 5 years. The median OS durations were 20.5 months [95\% confidence interval 
Table I. Comparison of clinicopathological factors by curability.

\section{\begin{tabular}{l} 
Characte \\
\hline Gender
\end{tabular}}

Male/female, $\mathrm{n}$

Age, year

Mean \pm SD

$59.3 \pm 8.6$

$66.7 \pm 10.2$

0.02

ECOG PS, n $(\%)$

0

$10(100)$

$34(72)$

0.10

$\geq 1$

$0(0)$

13 (28)

CEA, ng/ml ${ }^{\mathrm{a}}$

Normal/elevated $^{\mathrm{b}}, \mathrm{n}$

CA19-9, U/ml

Normal/elevated $^{\mathrm{c}}, \mathrm{n}$

Location of primary tumor ${ }^{\mathrm{d}}, \mathrm{n}(\%)$

Right-sided colon

Left-sided colon

Rectum

Histological differentiation, n (\%)

Well

Moderate

Undifferentiated

Unknown

Obstruction, $\mathrm{n}$

$$
\text { Absent/present }
$$

Maximum tumor diameter, $\mathrm{mm}^{\mathrm{a}}$

Mean \pm SD

Depth $^{\mathrm{a}}, \mathrm{n}$

Regional lymph node metastasis ${ }^{\mathrm{a}}, \mathrm{n}$

-pN1/pN2-

Preoperative CTX, $\mathrm{n}$

No/yes

Adjuvant CTX, n

No/yes

Postoperative $\mathrm{CTX}^{\mathrm{a}, \mathrm{e}}, \mathrm{n}$

$$
\text { No/yes }
$$

Lung metastases

Number, $\mathrm{n}$

Single/multiple

Laterality, $\mathrm{n}$

Unilateral/bilateral

Maximum diameter, $\mathrm{mm}$

$$
\text { Mean } \pm \text { SD }
$$

Hilar lymphadenopathy, $\mathrm{n}$

Extrapulmonary metastases ${ }^{\mathrm{f}}$ 
Table I. Continued.

\begin{tabular}{lccr}
\hline Characteristics & Curative $(\mathrm{n}=10)$ & Non-curative/stoma $(\mathrm{n}=47)$ & P-value \\
\hline Liver, n (\%) & $6(60)$ & $28(60)$ & 0.74 \\
Lymph nodes, n (\%) & $0(0)$ & $14(30)$ & 0.05 \\
Peritoneum, n (\%) & $0(0)$ & $8(17)$ & 0.33 \\
Bone, n (\%) & $0(0)$ & $4(9)$ & 1.00 \\
Adrenal gland, n (\%) & $0(0)$ & & 1.00 \\
Extrahepatic organs, & & $27 / 20$ & 0.01 \\
Absent/present & $10 / 0$ & & \\
\hline
\end{tabular}

${ }^{a}$ Excluding cases in which data were not available; ${ }^{\mathrm{b}} \mathrm{CEA}>5 \mathrm{ng} / \mathrm{ml} ;{ }^{\mathrm{c}} \mathrm{CA} 19-9>37 \mathrm{U} / \mathrm{ml} ;{ }^{\mathrm{d}}$ location of the more advanced lesion was counted in cases of multiple colorectal cancer: Including adjuvant chemotherapy; ${ }^{\mathrm{f}}$ multiple organs could be involved. SD, standard deviation; ECOG PS, Eastern Cooperative Oncology Group performance status; CEA, carcinoembryonic antigen; CA19-9, carbohydrate antigen 19-9; CTX, chemotherapy; N/A, not available.

Table II. Multivariate analysis for predictors of curative resection in colorectal cancer with lung metastases.

\begin{tabular}{lccc}
\hline Variable & Odds ratio & $95 \%$ CI & P-value \\
\hline $\begin{array}{l}\text { Age, years } \\
\geq 65\end{array}$ & 6.21 & $0.98-61.5$ & 0.052 \\
$<65$ & 1 & & \\
Obstruction & & & 0.070 \\
$\quad$ Present & 5.67 & $0.87-54.9$ & \\
Absent & 1 & & 0.081 \\
Lung metastases & & & \\
$\quad$ Bilateral & 4.93 & $0.82-33.4$ & \\
$\quad$ Unilateral & 1 & & 0.011 \\
Extrahepatic metastases & & & \\
Present & $1.74 \times 10^{7}$ & $2.0-\mathrm{N} / \mathrm{A}$ & \\
Absent & 1 & & \\
\hline
\end{tabular}

CI, confidence interval; N/A, not available.

(CI), 15.9-29.8 months] for the non-curative group and 7.9 months (95\% CI, 1.5-52.6 months) for the stoma group, respectively.

Finally, predictive factors for OS were analyzed in the 47 patients who underwent resection of the primary tumor. Univariate analysis revealed that bilateral lung metastases and non-curative resection were associated with an adverse survival rate. However, multivariate analyses using these parameters did not reveal any independent prognostic factor of OS (Table III).

\section{Discussion}

Metastasectomy is known to play a central role in improving the prognosis of patients with liver metastases from CRC (6). The reported 5-year survival rate ranged 39-51\% after hepatectomy (9-11). Additionally, perioperative systemic chemotherapy appears to reduce the risk of relapse in addition to surgical resection for CRC with liver-limited disease. The European Organisation for Research and Treatment of Cancer (EORTC) intergroup trial 40983, a milestone phase III randomized control study, demonstrated that 6 additional courses of FOLFOX before and after hepatectomy improved relapse-free survival in $\mathrm{CRC}$ with initially resectable liver metastases by 7.4 months (7).

The efficacy of surgical resection for lung metastases from CRC has been demonstrated in several institutions. The 3 -year survival rate after pulmonary metastasectomy ranged from 53 to $80 \%$ and the 5-year survival rate was reported to be $37-68 \%$ (12-17). However, subjects in these studies had synchronous and metachronous lung metastases $(12,13,15)$. Furthermore, non-surgical therapies for lung metastases were not discussed in the majority of them; only a few retrospective studies demonstrated that radical lung metastasectomy produced a median survival advantage of 37-41 months over non-surgical cases $(18,19)$. In this regard, standard treatment strategies have not been fully investigated for CRC with lung metastases. The present study was characterized by CRC patients with exclusively synchronous lung metastases to whom various treatment modalities had been applied. To the best of our knowledge, this is the first study of the predictive factors for curative resection of CRC with synchronous lung metastases.

In the study, an age $>65$ years, obstructive primary tumor, bilateral lung involvement and extrahepatic disease were associated with non-curative surgery for CRC accompanied by lung metastases. Of these, extrahepatic disease was identified as an independent predictor by multivariate analysis. Therefore, carefully selected patients with synchronous metastases confined to the lung and liver can be candidates for radical surgery. Previous studies projected a 5-year survival of $25-74 \%$ following liver and lung resections for metastases from CRC in selected patients (17,20-23), which were similar to the surgical outcomes for CRC with liver-only or lung-only metastases. Consistent with previous findings $(18,19)$, patients who underwent curative resections achieved a markedly longer OS than those who underwent palliative therapies in the present study (Fig. 2). Notably, 4 out of 5 long survivors 
Table III. Univariate and multivariate analyses for predictors of the overall survival rate in patients undergoing tumor resection.

\begin{tabular}{|c|c|c|c|c|c|c|}
\hline \multirow[b]{2}{*}{ Variable } & \multicolumn{3}{|c|}{ Univariate analysis } & \multicolumn{3}{|c|}{ Multivariate analysis } \\
\hline & HR & $95 \% \mathrm{CI}$ & P-value & HR & $95 \% \mathrm{CI}$ & P-value \\
\hline Age, $<65$ vs. $\geq 65$ years & 1.22 & $0.54-2.77$ & 0.628 & & & \\
\hline Gender, female vs. male & 0.61 & $0.23-1.41$ & 0.256 & & & \\
\hline PS, poor vs. 0 & 1.19 & $0.34-3.23$ & 0.761 & & & \\
\hline Normal CEA vs. elevated $^{\mathrm{a}}$ & 1.30 & $0.47-3.12$ & 0.584 & & & \\
\hline Normal CA19-9 vs. elevated ${ }^{b}$ & 0.95 & $0.42-2.21$ & 0.897 & & & \\
\hline Colon vs. rectal cancer & 1.16 & $0.49-2.67$ & 0.728 & & & \\
\hline Well-differentiated histology vs. others & 0.66 & $0.24-1.59$ & 0.368 & & & \\
\hline Obstruction vs. none & 1.05 & $0.46-2.41$ & 0.915 & & & \\
\hline Primary tumor size, $<50$ vs. $\geq 50 \mathrm{~mm}$ & 1.08 & $0.48-2.44$ & 0.859 & & & \\
\hline pT4 vs. $\leq$ pT3 & 1.70 & $0.74-3.97$ & 0.210 & & & \\
\hline pN2-3 vs. pN0-1 & 1.70 & $0.70-3.97$ & 0.235 & & & \\
\hline Lung metastasis, $>10$ vs. $\leq 10 \mathrm{~mm}$ & 0.53 & $0.22-1.20$ & 0.128 & & & \\
\hline Single vs. multiple lung metastasis & 0.39 & $0.06-1.34$ & 0.152 & & & \\
\hline Unilateral vs. bilateral lung metastases & 0.37 & $0.11-0.98$ & 0.048 & 0.69 & $0.17-2.17$ & 0.545 \\
\hline Hilar lymphadenopathy vs. none & 0.93 & $0.14-3.22$ & 0.922 & & & \\
\hline Extrahepatic disease vs. none & 0.53 & $0.19-1.64$ & 0.245 & & & \\
\hline Curative vs. non-curative surgery & 0.23 & $0.05-0.72$ & 0.009 & 0.30 & $0.06-1.11$ & 0.074 \\
\hline Preoperative CTX vs. none & 1.55 & $0.36-4.63$ & 0.504 & & & \\
\hline Adjuvant CTX vs. none & 0.27 & $0.01-2.88$ & 0.273 & & & \\
\hline Postoperative CTX vs. none & 1.28 & $0.43-5.50$ & 0.683 & & & \\
\hline
\end{tabular}

${ }^{\mathrm{a} C E A}>5 \mathrm{ng} / \mathrm{ml}$; ${ }^{\mathrm{b}} \mathrm{CA} 19-9>37 \mathrm{U} / \mathrm{ml}$. HR, hazard ratio; CI, confidence interval; PS, performance status; CEA, carcinoembryonic antigen; CA19-9, carbohydrate antigen 19-9; CTX, chemotherapy.

underwent repeated surgery and 1 received radiation. In addition, postoperative chemotherapy was administered to $\sim 80 \%$ regardless of curability, underscoring the importance of a multidisciplinary approach to overcome recurrence.

We previously suggested the possible benefits of non-surgical treatments, including chemotherapy, in patients with unresectable lung metastases from CRC by reviewing improvements in their prognoses over time (24). In addition, a recent cohort study reported the positive impact of chemotherapy on survival in CRC with synchronous lung metastases regardless of surgery (19). In the new era of chemotherapy, the effects of perioperative chemotherapy on the surgical outcome in CRC patients with resectable lung metastases are worth investigating by conducting clinical trials similar to the EORTC intergroup 40983.

Palliative resection of primary CRC provided improved prognoses than stoma surgery alone in the present cohort (Fig. 2). Resection of the primary tumor in incurable stage IV disease is indicated in order to relieve an obstruction, pain, bleeding and other symptoms or to prevent perforation. A reduction in the tumor burden may allow for a better response to subsequent systemic chemotherapy (25). By contrast, complications following major surgery may delay the initiation of systemic chemotherapy in incurable CRC (26). A meta-analysis estimated that palliative resection of stage IV CRC could improve in OS by 6 months with acceptable postoperative morbidity (27). However, another meta-analysis revealed the absence of consistent benefits by surgery in non-curative stage IV CRC if asymptomatic (28). This discrepancy will be addressed by the results of an ongoing randomized controlled 'SYNCHRONOUS' study comparing resection and non-resection of the primary site in unresectable CRC cases (29).

The present study is limited by several factors. First, it is retrospective in nature and there were potential selection biases in performing metastasectomy, resection of primary CRC and stoma surgery. Patients who received non-surgical treatments alone were not included in the cohort. Second, there were no definite criteria for preoperative and postoperative chemotherapies. Whether to prescribe chemotherapy partly depended on the individual PS to withstand the treatment and the perceived benefits. Another limitation was low patient numbers, similar to those in previous studies of lung metastases from CRC, which were partly due to the focus on synchronous lung metastases.

In conclusion, for the first time, the absence of extrahepatic metastases was demonstrated as an independent predictor of curative surgery in CRC patients with synchronous lung metastases. The selected patients who underwent pulmonary metastasectomy may achieve a longer survival rate by meticulous postoperative therapies.

\section{Acknowledgements}

The present study was supported by the Ministry of Education, Culture, Sports, Science and Technology of Japan and by the Ministry of Health, Labor and Welfare of Japan. 


\section{References}

1. Siegel R, Ma J, Zou Z and Jemal A: Cancer statistics, 2014. CA Cancer J Clin 64: 9-29, 2014.

2. Ferlay J, Steliarova-Foucher E, Lortet-Tieulent J, Rosso S, Coebergh JW, Comber H, Forman D and Bray F: Cancer incidence and mortality patterns in Europe: Estimates for 40 countries in 2012. Eur J Cancer 49: 1374-1403, 2013.

3. Matsuda A, Matsuda T, Shibata A, et al: Cancer incidence and incidence rates in Japan in 2007: A study of 21 population-based cancer registries for the Monitoring of Cancer Incidence in Japan (MCIJ) project. Jpn J Clin Oncol 43: 328-336, 2013.

4. Penna C and Nordlinger B: Colorectal metastasis (liver and lung). Surg Clin North Am 82: 1075-1090, 2002.

5. Van Cutsem E, Nordlinger B and Cervantes A: Advanced colorectal cancer: ESMO Clinical Practice Guidelines for treatment. Ann Oncol 21 (Suppl 5): v93-v97, 2010.

6. Mahmoud N and Bullard Dunn K: Metastasectomy for stage IV colorectal cancer. Dis Colon Rectum 53: 1080-1092, 2010.

7. Nordlinger B, Sorbye H, Glimelius B, et al: Perioperative FOLFOX 4 chemotherapy and surgery versus surgery alone for resectable liver metastases from colorectal cancer (EORTC 40983): Long-term results of a randomised, controlled, phase 3 trial. Lancet Oncol 14: 1208-1215, 2013.

8. Sobin LH, Gospodarowicz MK and Wittekind C (eds). TNM Classification of Malignant Tumors. 7th edition. John Wiley \& Sons Inc., New Jersey, NJ, pp100-105, 2009.

9. Wei AC, Greig PD, Grant D, Taylor B, Langer B and Gallinger S: Survival after hepatic resection for colorectal metastases: A 10-year experience. Ann Surg Oncol 13: 668-676, 2006.

10. Giuliante F, Ardito F, Vellone M, Ranucci G, Federico B, Giovannini I and Nuzzo G: Role of the surgeon as a variable in long-term survival after liver resection for colorectal metastases. J Surg Oncol 100: 538-545, 2009.

11. Saiura A, Yamamoto J, Hasegawa K, Koga R, Sakamoto Y, Hata S, Makuuchi M and Kokudo N: Liver resection for multiple colorectal liver metastases with surgery up-front approach: Bi-institutional analysis of 736 consecutive cases. World J Surg 36: 2171-2178, 2012.

12. Yedibela S, Klein P, Feuchter K, Hoffmann M, Meyer T, Papadopoulos T, Göhl J and Hohenberger W: Surgical management of pulmonary metastases from colorectal cancer in 153 patients. Ann Surg Oncol 13: 1538-1544, 2006.

13. Watanabe K, Nagai K, Kobayashi A, Sugito M and Saito N: Factors influencing survival after complete resection of pulmonary metastases from colorectal cancer. Br J Surg 96: 1058-1065, 2009.

14. Onaitis MW, Petersen RP, Haney JC, et al: Prognostic factors for recurrence after pulmonary resection of colorectal cancer metastases. Ann Thorac Surg 87: 1684-1688, 2009.

15. Rama N, Monteiro A, Bernardo JE, Eugénio L and Antunes MJ: Lung metastases from colorectal cancer: Surgical resection and prognostic factors. Eur J Cardiothorac Surg 35: 444-449, 2009.

16. Mitry E, Guiu B, Cosconea S, et al: Epidemiology, management and prognosis of colorectal cancer with lung metastases: A 30-year population-based study. Gut 59: 1383-1388, 2010.

17. Hattori N, Kanemitsu Y, Komori K, Shimizu Y, Sano T, Senda Y, Mitsudomi T and Fukui T: Outcomes after hepatic and pulmonary metastasectomies compared with pulmonary metastasectomy alone in patients with colorectal cancer metastasis to liver and lungs. World J Surg 37: 1315-1321, 2013.
18. Tampellini M, Ottone A, Bellini E, et al: The role of lung metastasis resection in improving outcome of colorectal cancer patients: Results from a large retrospective study. Oncologist 17: 1430-1438, 2012.

19. Nordholm-Carstensen A, Krarup PM, Jorgensen LN Wille-Jørgensen PA and Harling H: Occurrence and survival of synchronous pulmonary metastases in colorectal cancer: A nationwide cohort study. Eur J Cancer 50: 447-456, 2014.

20. Miller G, Biernacki P, Kemeny NE, Gonen M, Downey R, Jarnagin WR, D'Angelica M, Fong Y, Blumgart LH and DeMatteo RP: Outcomes after resection of synchronous or metachronous hepatic and pulmonary colorectal metastases. J Am Coll Surg 205: 231-238, 2007.

21. Brouquet A, Vauthey JN, Contreras CM, Walsh GL, Vaporciyan AA, Swisher SG, Curley SA, Mehran RJ and Abdalla EK: Improved survival after resection of liver and lung colorectal metastases compared with liver-only metastases: A study of 112 patients with limited lung metastatic disease. J Am Coll Surg 213: 62-71, 2011.

22. Schüle S, Dittmar Y, Knösel T, Krieg P, Albrecht R, Settmacher U and Altendorf-Hofmann A: Long-term results and prognostic factors after resection of hepatic and pulmonary metastases of colorectal cancer. Int J Colorectal Dis 28: 537-545, 2013.

23. Tsukamoto S, Kinugasa Y, Yamaguchi T and Shiomi A: Survival after resection of liver and lung colorectal metastases in the era of modern multidisciplinary therapy. Int J Colorectal Dis 29: 81-87, 2014

24. Nozawa H, Sunami E, Nakajima J, Nagawa H and Kitayama J: Synchronous and metachronous lung metastases in patients with colorectal cancer: A 20-year monocentric experience. Exp Ther Med 3: 449-456, 2012

25. Galizia G, Lieto E, Orditura M, Castellano P, Imperatore V, Pinto $\mathrm{M}$ and Zamboli A: First-line chemotherapy vs bowel tumor resection plus chemotherapy for patients with unresectable synchronous colorectal hepatic metastases. Arch Surg 143: 352-358, 2008.

26. Benoist S, Pautrat K, Mitry E, Rougier P, Penna C and Nordlinger B: Treatment strategy for patients with colorectal cancer and synchronous irresectable liver metastases. $\mathrm{Br}$ J Surg 92: 1155-1160, 2005.

27. Stillwell AP, Buettner PG and Ho YH: Meta-analysis of survival of patients with stage IV colorectal cancer managed with surgical resection versus chemotherapy alone. World J Surg 34: 797-807, 2010.

28. Cirocchi R, Trastulli S, Abraha I, Vettoretto N, Boselli C, Montedori A, Parisi A, Noya G and Platell C: Non-resection versus resection for an asymptomatic primary tumour in patients with unresectable stage IV colorectal cancer. Cochrane Database Syst Rev 8: CD008997, 2012.

29. Rahbari NN, Lordick F, Fink C, et al: Resection of the primary tumour versus no resection prior to systemic therapy in patients with colon cancer and synchronous unresectable metastases (UICC stage IV): SYNCHRONOUS - a randomised controlled multicentre trial (ISRCTN30964555). BMC Cancer 12: 142, 2012. 